\title{
Proposal for Driver Distraction Indexes Using Biological Signals Including Eye Tracking
}

\author{
Nobumichi Takahashi ${ }^{1}$, Satoshi Inoue ${ }^{1}$, Hironori Seki ${ }^{1}$, Shuhei Ushio ${ }^{1}$, Yukou Saito ${ }^{2}$, \\ Koyo Hasegawa $^{2}$, and Michiko Ohkura ${ }^{1}$ \\ ${ }^{1}$ Shibaura Institute of Technology, 3-7-5, Toyosu, Koto-ku, Tokyo,135-8548, Japan \\ \{ma12066,109011,109057,109013, ohkura\} ashibaura-it.ac.jp \\ ${ }^{2}$ Alpine Electronics Inc, 1-7, yukigayaotsuka-machi, Ota-ku, Tokyo, 145-8501, Japan \\ \{yukou-saito,k-hasegawa\} @apn.alpine.co.jp
}

\begin{abstract}
According to the AAA Foundation for Traffic Safety, driver inattention is a major contributor to highway crashes. Above all, driver distraction is an important factor. As a result, many studies have been performed on it. We also performed experiments on candidates for biological indexes. In this paper, we employed new biological signals (eye tracking). Then, we performed an experiment to find new candidates for biological indexes. We obtained new knowledge from the result of that experiment.
\end{abstract}

Keywords: Driver distraction, Biological signal.

\section{Introduction}

According to the AAA Foundation for Traffic Safety [1], driver inattention is a major contributor to highway crashes. The National Highway Traffic Safety Administration estimates that approximately $25 \%$ of police-reported crashes involve some form of driver inattention, where the driver is distracted, asleep, fatigued, or "lost in thought" [2]. Estimates from other sources are as high as 35-50\%.

Against these backgrounds, much research has been performed on driver distraction [3]. We also performed experiments to discover candidates for biological indexes of driver distraction, shown in Table 1, and found some useful ones.

Table 1. Candidates for biological indexes

\begin{tabular}{cc}
\hline Biosignals & Biological Indexes \\
\hline ECG & RRI, heart rate, LF/HF \\
\hline (Electrocardiogram $)$ & Power spectrum of alpha, beta, delta waves, \\
EEG & ratio of each wave, \\
\hline (Electroencephalogram) & SPR, SPL \\
\hline SPA (Galvanic Skin Potential) & \\
\hline
\end{tabular}


Then, we performed a new experiment employing eye-tracking data to find new candidates for biological indexes as the next step.

This article describes the experiment, its results, and the proposal of useful biological indexes for driver distraction.

\section{Experimental System Including Driving Simulator}

An outline of our experimental system is shown in Fig 1. The system consists of a driving simulator (DS) and measurement systems for biological signals. The driving simulator consists of the following components:

- Display(s)

- Speakers

- Wheel

- Pedals

- Sheet

- Radio channel tuning system(s)

- PC(s) to control simulator operation.

The measurement system consists of the following components:

- Sensors (or electrodes) and instruments to measure ECG, SPA and eye tracking

- PCs to control the measurement of these biological signals

To measure SPA, the palmar surface of the finger is commonly used. However, since palms are involved in holding steering wheels, we instead employed the bottom of the foot. Figure 2 shows a diagram of the eye-tracking system.

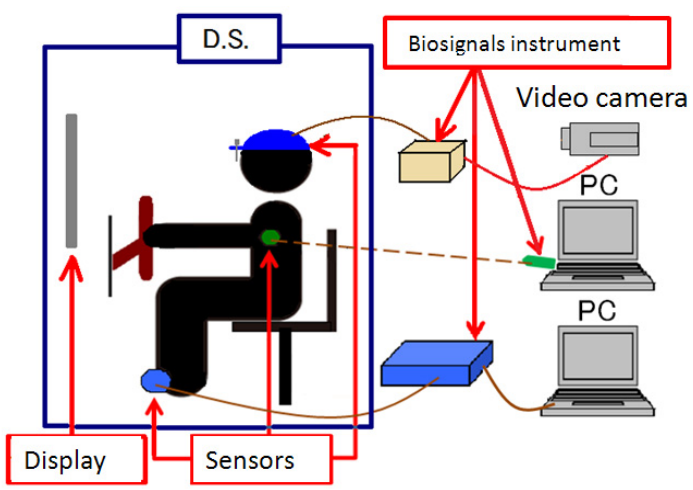

Fig. 1. Outline of experimental system 


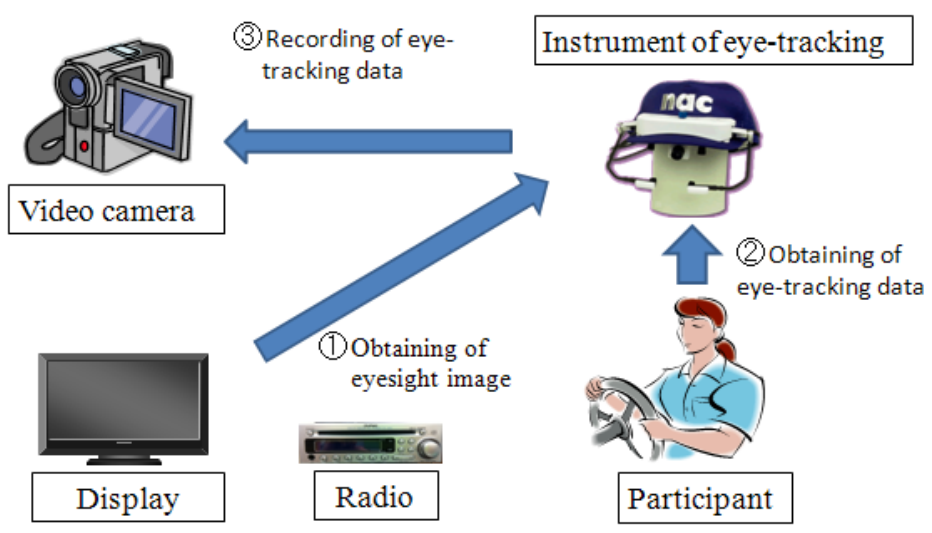

Fig. 2. Diagram of eye-tracking system

\section{Experimental Method}

\subsection{Experimental Purpose}

The purposes of this experiment are as follows:

- to find some candidates of indexes for eye-tracking data.

- - to obtaining new indexes from combinations of biological indexes.

\subsection{Test Course}

The test course was a straight line with three lanes and flags set randomly to show the lanes for lane changes (Fig. 3). It takes three minutes to drive this course at $100 \mathrm{~km} / \mathrm{h}$.

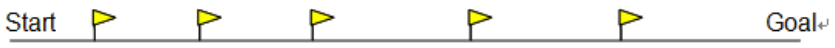

Fig. 3. Test course with flags

\subsection{Experimental Task}

The main task was the driving task with lane change. The secondary task was radio channel selection. An ordinary car radio was employed, and the frequencies to be set were orally given by the experimenter.

The kinds of experimental tasks are as follows:

1. Driving (main task only)

2. Interval A (main task + secondary task with an interval of 2 seconds)

3. Interval B (main task + secondary task with changing lanes through flags 2 times)

4. Interval $\mathrm{C}$ (main task + secondary task with changing lanes through flags 3 times) 


\section{$3.4 \quad$ D. D. Indexes}

We employed biological indexes such as RRI, SPR, and stationary point for the D. D. indexes. RRI means an interval between R-wave and R-wave of ECG. SPR means change of resistance of skin by temporary sweating.

\section{Experimental Results}

\subsection{Experimental}

We performed the experiment with four volunteers in their 20s. The experiment took about $1.5 \mathrm{~h}$ per participant. Each participant repeated each task four times. The setup of the driving simulator is shown in Fig. 4.

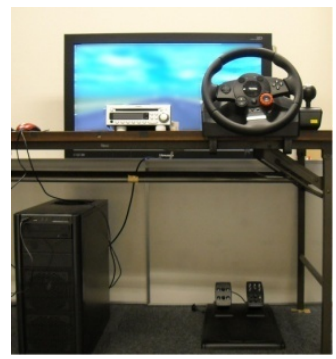

Fig. 4. Appearance of driving simulator

\subsection{Experimental Results of Eye Tracking}

We considered indexes of eye tracking for candidates as follows:

- -stationary point

- -steady gaze at the radio

- -gaze-movement speed

- -gaze-movement amount

- -distance of gaze

- -pupil diameter

We will describe especially useful candidates.

- Gaze-movement speed

Figure 5 shows the average gaze-movement speed. We performed a two-factor analysis of variance and multiple comparison between each task. Both main effects of tasks and participants were effective at $1 \%$ and $5 \%$ levels. The results of multiple comparison are as follows:

Driving $<$ Interval A, B, C $(\mathrm{p}<0.01)$

Interval $\mathrm{B}, \mathrm{C}<$ Interval A $(\mathrm{p}<0.01)$ 


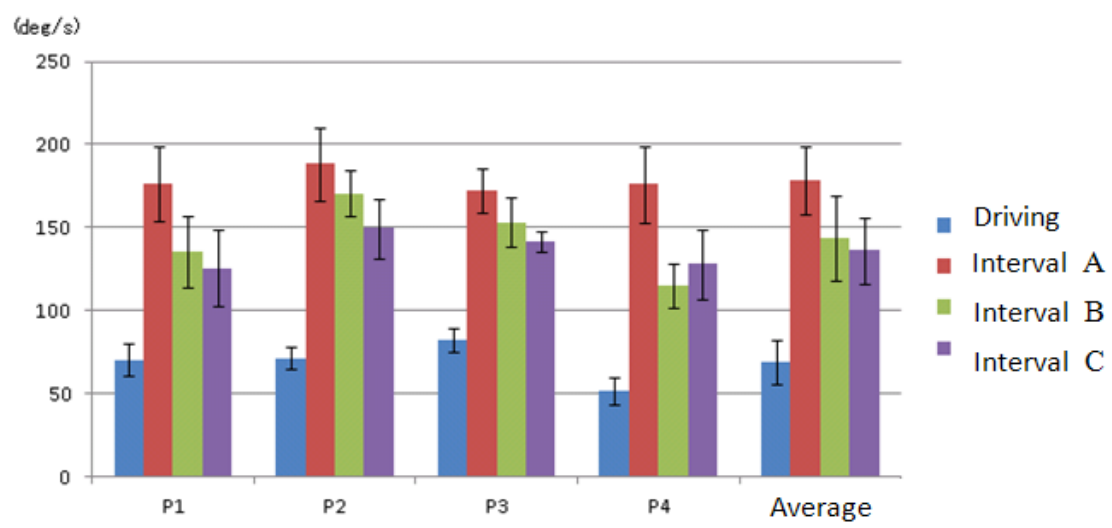

Fig. 5. Averaged gaze-movement speed

\section{- Pupil diameter}

Figure 6 shows average pupil diameter of subjects' left eyes. We performed a twofactor analysis of variance and multiple comparison between each task. Both main effects of participants and tasks were effective at $1 \%$ and 5\% levels. The results of multiple comparison are as follows:

\section{Driving $<$ Interval A $(\mathrm{p}<0.01)$}

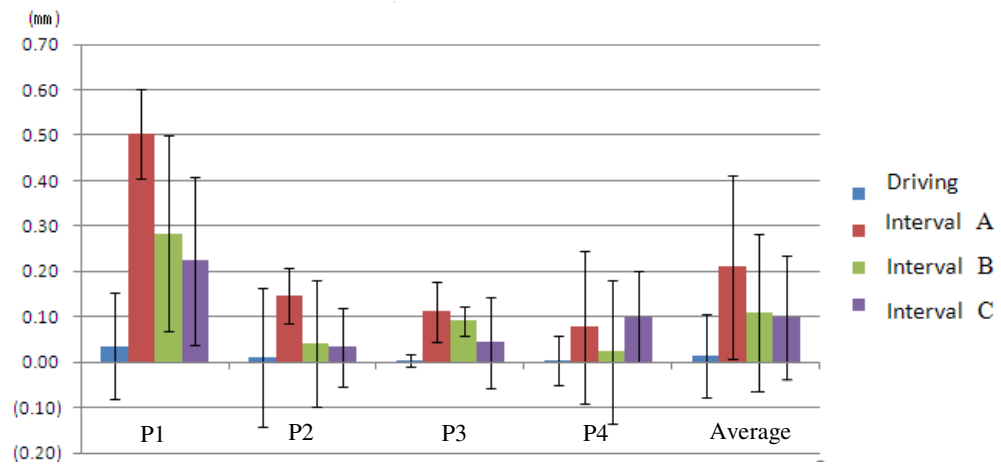

Fig. 6. Average pupil diameter of left eye

\subsection{Experimental Results of ECG and SPA}

Result of ECG and SPA indicates a similar tendency to previous research as follows [3].

- At ECG, RRI is very appropriate for detecting the difference between each task. It indicates mental work-load. 
- At SPA, average of SPR is also appropriate for detecting the difference between each task. It indicates nervousness of the driver.

\subsection{Experimental Results of Combination of Indexes}

We performed correlation analysis to indexes that we measured in this experiment. We will describe useful combinations.

- $\quad$ RRI and steady gaze at radio

At RRI, there's no statistical difference between Intervals B and C. However, combination of RRI and steady gaze at radio has a difference between these tasks. $(r=-$ $0.543, \mathrm{P}<0.01)$

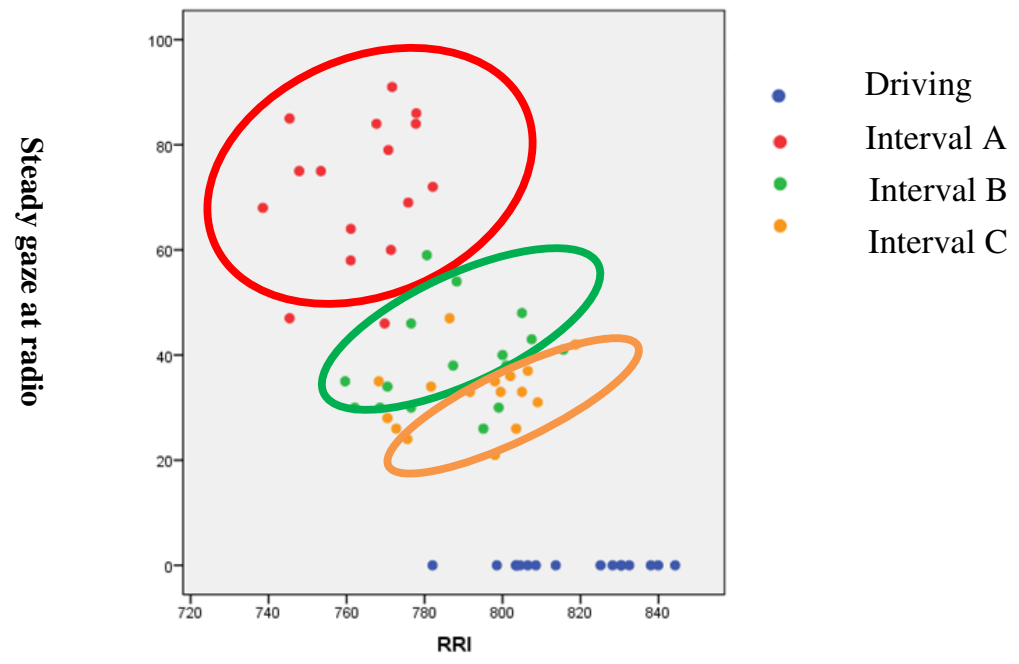

Fig. 7. Results of combination of RRI and Steady gaze of radio

\subsection{Redefinition of Start of Secondary Task}

In previous research [4], we defined the start of the secondary task as when the experimenter showed the task to the participant. However, we redefined the start of the secondary task more strictly using eye tracking.

\section{Discussion}

RRI and SPA showed a similar tendency to previous research. This means those are reliable indexes for measuring D.D. At some indexes for eye tracking, there's a statistical difference between tasks. This indicates those indexes could be indexes for D.D. However, we need further experiments to confirm the reliability of those indexes. 


\section{Conclusions}

We performed an experiment and obtained the following conclusions.

- RRI and SPA are reliable indexes for D. D.

- Gaze-movement speed and pupil diameter may become indexes of D. D.

- From the result of combination of indexes, RRI and steady gaze at radio shows new possibility.

We must perform further experiments to confirm these new indexes.

\section{References}

1. Stutts, J., et al.: The Role of Driver Distraction in Traffic Crashes. In: AAA Foundation for Traffic Safety, Washington DC (2001)

2. Ranney, T., Garrott, W., Goodman, M.: NHTSA Driver Distraction Research: Past, Present, and Future. In: NHTSA, Washington DC (2000)

3. Young, K., Lee, J., Regan, M.: Driver Distraction: Theory, Effects, and Mitigation. CRC Press, New York (2008)

4. Ohkura, M., et al.: Proposal for driver distraction indexes using biological signals. In: ASME International Design Engineering Technical Conference \& Computers and Information in Engineering Conference, Chicago (2012) 